Management international

International Management

Gestiòn Internacional

\title{
Sélection des rédacteurs invités
}

\section{Frank Brulhart}

Volume 23, numéro 4, 2019

URI : https://id.erudit.org/iderudit/1066081ar

DOI : https://doi.org/10.7202/1066081ar

Aller au sommaire du numéro

Éditeur(s)

HEC Montréal

Université Paris Dauphine

ISSN

1206-1697 (imprimé)

1918-9222 (numérique)

Découvrir la revue

Citer ce compte rendu

Brulhart, F. (2019). Compte rendu de [Sélection des rédacteurs invités].

Management international / International Management / Gestiòn Internacional, 23(4), 193-193. https://doi.org/10.7202/1066081ar

Tous droits réservés (C) Management international / International Management / Gestión Internacional, 2019
Ce document est protégé par la loi sur le droit d'auteur. L’utilisation des services d'Érudit (y compris la reproduction) est assujettie à sa politique d'utilisation que vous pouvez consulter en ligne.

https://apropos.erudit.org/fr/usagers/politique-dutilisation/ 
Titre de l'ouvrage : Révolution du management des ressources humaines. Des compétences aux capabilités

Date de publication : 2016

Éditeur : Presses Universitaires du Septentrion, Villeneuve d'Ascq.

Coordinateurs : Solveig Fernagu Oudet et Christian Bayal

La question posée dans cet ouvrage collectif est fondamentalement celle du sens et de l'usage du terme de compétences. Pour cela, les auteurs proposent 3 parties qui regroupent 29 chapitres. La première partie revient sur l'origine de la compétence ainsi que son caractère polysémique, en partie lié à l'évolution même de sa conception. Dans la seconde partie de l'ouvrage les chapitres proposent une vision critique de la notion de gestion des compétences notamment en termes d'évaluation des compétences et de systèmes de formation. Enfin dans la troisième partie, les auteurs proposent de substituer au terme de compétence, qu'ils considèrent comme désormais inadapté, la notion de «capabilité». Adoptant une vision multidisciplinaire, l'ouvrage propose la capabilité comme le moyen d'envisager les compétences et les moyens de les mettre en œuvre et de les développer. Pour cela, les auteurs convoquent la notion d'environnements capacitants, en tant que condition présupposée d'un réel développement des individus au travail.

Titre de l'ouvrage : Activités et collectifs. Approches cognitives et organisationnelles

Date de publication : A paraître

Editeur : Presses Universitaires de Provence, Aix en Provence Coordinateurs : Lise Gastaldi, Cathy Krohmer et Claude Paraponaris

Cet ouvrage collectif est le fruit d'un travail pluridisciplinaire de plusieurs universitaires. Il traite des questions suivantes: la dynamique des organisations et des entreprises, l'apprentissage individuel et collectif, la prise de décision en contexte d'activité, les nouvelles formes d'organisation entre marchand et non-marchand, la logistique et la place de l'expert dans l'entreprise. Onze chapitres font état de travaux conduits dans des organisations de nature variée : entreprises industrielles, municipalités, encyclopédie Wikipédia, espaces de coworking, chaînes logistiques, projets, etc. Ces différentes perspectives de recherche témoignent de la richesse de l'apport des approches cognitives à l'analyse des activités et des collectifs en se focalisant, non pas sur les règles et routines en vigueur au sein des organisations, mais sur les mouvements émergents qui tendent à redéfinir ce qu'est un collectif, ce que suppose un apprentissage de nouvelles règles d'action et, en définitive, comment se transforment les activités de travail dans des contextes dominés par des enjeux d'innovation intensive.
Titre de l'ouvrage : Management des compétences - Enjeux, modèles et perspectives

Date de publication : 2016

Editeur : Dunod, Paris

Auteurs : Anne Dietrich, Patrick Gilbert, Frédérique Pigeyre, Jacques Aubret, (2010) et Dunod

30 ans après le développement du concept de compétence dans le champ des sciences de gestion, cet ouvrage a pour ambition de proposer un état des lieux des connaissances sur ce thème et sur la question du management des compétences. Pour cela les auteurs abordent les enjeux et la diversité de la gestion des compétences, les expérimentations liées au management des compétences et les débats qu'elles ont pu générer ainsi que les démarches compétences, en tant qu'analyseur des formes de management. L'ouvrage se décompose en trois parties principales. La première met l'accent sur le caractère contingent de la gestion des compétences et propose un cadre d'analyse favorisant la compréhension et la mise en œuvre des démarches compétences. La deuxième met en avant les imbrications fonctionnelles dans les pratiques de management des compétences. La troisième illustre les problèmes récurrents liés au management des compétences en mobilisant des cas. Enfin l'ouvrage conclut sur la contribution des démarches compétences à l'amélioration des pratiques de management. 